\title{
On uncertainty principle of the local polynomial Fourier transform
}

Xiumei $\mathrm{Li}^{1}$, Guoan $\mathrm{Bi}^{\mathrm{i}^{*}}$ and Shenghong $\mathrm{Li}^{3}$

\begin{abstract}
In this article, a comprehensive study on uncertainty principle of the local polynomial Fourier transform (LPFT) is presented. It shows that the uncertainty product of the LPFT of an arbitrary order is related to the parameters of the signal and the window function, in addition to the errors of estimating the polynomial coefficients. Important factors that affect resolutions of signal representation, such as the window width, the length of overlap between signal segments, order mismatch and estimation errors of polynomial coefficients, are discussed. The effects of minimizing computational complexities on signal representation by reducing the order of the transform and the overlap length between signal segments are also examined. In terms of the signal concentration, comparisons among the short-time Fourier transform, the Wigner-Ville distribution and the second order LPFT are presented. The LPFT is shown to be an excellent candidate providing better representations for time-varying signals.
\end{abstract}

Keywords: uncertainty principle, short-time Fourier transform, local polynomial Fourier transform, Wigner-Ville distribution

\section{Introduction}

The Fourier transform is an important tool to obtain signal representation in the frequency domain. However, it is not suitable to deal with time-varying signals that contain frequencies changing with time. To describe the frequency characteristics of such signals, the short-time Fourier transform (STFT), defined as

$$
\operatorname{STFT}(t, \omega)=\int_{-\infty}^{+\infty} s(\tau) h(\tau-t) \exp \{-j \omega \tau\} d \tau,
$$

is widely used. ${ }^{a}$ The basic assumption of the STFT is that the frequencies in each signal segment are not changed with time so that the frequency variations with time are approximately described by the Fourier transforms of the successive signal segments. Because this assumption is not generally true, however, the resolution of the STFT in the time-frequency domain is often limited.

To overcome this drawback, other time-frequency transforms such as fractional Fourier transform $[1,2]$

\footnotetext{
* Correspondence: egbi@ntu.edu.sg

${ }^{2} \mathrm{School}$ of Electrical and Electronic Engineering, Nanyang Technological University, Singapore

Full list of author information is available at the end of the article
}

and local polynomial Fourier transform (LPFT) [3] were proposed. As a generalization of the STFT, the kernel of the LPFT uses extra parameters to approximate the phase of the signal into a polynomial form. With these parameters, or the polynomial coefficients, the LPFT can describe the time-varying frequencies with a better accuracy, and therefore the resolution of signal representation in the time-frequency domain can be significantly improved compared with that achieved by the STFT. Because the LPFT is a linear transform, it is free from the cross terms which may exist in some other time-frequency representations. Due to its desirable advantages, the LPFT has found applications in various areas. For example, it has been used to improve radar images of fast maneuvering targets [4,5]. It has been applied for non-stationary interference suppression in noise radar systems [6], as well as in spread spectrum communications [7], to achieve improved performance compared with that obtained by the STFT. In [8], the LPFT-based beamformer is derived for source localization and tracking in nonstationary environment, which can resolve closely spaced sources when their velocities are sufficiently different. Moreover, the LPFT can be used as an instantaneous frequency estimator, and the corresponding asymptotic covariance matrix and bias of 
the estimates are studied in [3]. A comprehensive review on the LPFT and its applications can be referred to [9].

In the classical time-frequency domain, the product of time duration and bandwidth cannot be arbitrarily small and is generally described in the form of the uncertainty principle [10]. The uncertainty principle plays an important role in signal analysis [10], and studies on the uncertainty principle of various transforms have been reported in the literature [1,11-16]. For example, it was reported that the STFT is limited by the uncertainty principle [17]. The local uncertainty principle was demonstrated in [12] by defining local quantities as conditional standard deviations, while the standard formulation of the uncertainty principle is in terms of global standard deviation. It shows that the local uncertainty product of the spectrogram indeed has a lower bound due to the windowing approach but it is not a property of the signal or a fundamental limit. For a large class of bilinear time-frequency distributions, the local uncertainty product is always less than or equal to the global uncertainty product and can be arbitrarily small [12].

It has been observed that the resolution of the LPFT in the time-frequency domain is also influenced by the window length which controls the tradeoff of bias and variance $[3,18]$. Therefore, it is believed that there must exist some form of the uncertainty principle based on the LPFT. The uncertainty principle of the second order LPFT was only recently discussed in [19]. It was shown that the uncertainty product of the second order LPFT for the second order PPS is time-independent when Gaussian window is used to segment the signal. However, a comprehensive study on the uncertainty principle for an arbitrary order LPFT has not been reported in the literature.

Similar to the steps used to derive the uncertainty principle of the STFT [17], this article firstly reports the mathematical derivations of the uncertainty principles of the second, third, fourth order and thereafter $M$ th order LPFTs. It is found that the uncertainty product of an arbitrary order LPFT is related to the parameters of the signal and the window function, as well as the errors of estimating the polynomial coefficients. When the polynomial coefficients of the transform kernel are accurately estimated, the uncertainty product becomes a constant if a Gaussian window is used to segment the input signal. Based on the derived signal duration and bandwidth, we can obtain the uncertainty product. Then we will discuss the effects, in terms of the resolution in the time-frequency domain and the minimization of the required computational complexity, of window width, order mismatch, i.e., using the second order LPFT to process higher-order PPS, and the length of overlap between signal segments. Comparisons are also made on the resolutions of signal representations in the time- frequency domain achieved by using the STFT, the Wigner-Ville distribution (WVD) and the second order LPFT.

The rest of the article is organized as follows. With the similar terminologies used in deriving the uncertainty principle of the STFT in Appendix [17], the uncertainty principles of the LPFTs of various orders are given in Section 2. Section 3 discusses the effects of various issues on obtaining desirable signal representations in the time-frequency domain. Signal concentrations of different time-frequency representations are also compared. Finally conclusions are given in Section 4.

\section{Uncertainty principles of the LPFTs}

In this section, the definition of the LPFT is given and some important issues that affect the computational complexity are addressed. Then we will give the derivation of the uncertainty principles of different order LPFTs and achieve the general expression of the uncertainty principle for the Mth order LPFT.

\subsection{The LPFT}

The LPFT is a generalization of the STFT, and the $M$ thorder LPFT is defined as $[3,9]$ :

$$
\begin{aligned}
\operatorname{LPFT}(t, \omega) & =\int s(t+\tau) h(\tau) \exp \left\{-j \omega \tau-j \sum_{m=2}^{M} \frac{\omega_{m-1} \tau^{m}}{m !}\right\} d \tau, \\
& =\int s(\tau) \exp \left\{j \omega t-j \sum_{m=2}^{M} \frac{\omega_{m-1}(\tau-t)^{m}}{m !}\right\} h(\tau-t) \exp \{-j \omega \tau\} d \tau,
\end{aligned}
$$

where $M$ is the order of the polynomial function, $\omega_{2}=\frac{d^{2} \Omega(t)}{d t^{2}}, \omega_{2}=\frac{d^{2} \Omega(t)}{d t^{2}}, .$. , and $\omega_{M-1}=\frac{d^{M-1} \Omega(t)}{d t^{M-1}}$ are the polynomial coefficients, and $\Omega(t)$ is the instantaneous frequency of the signal.

The LPFT is particularly suited to process the polynomial phase signals (PPSs) with a Gaussian amplitude defined by

$$
s(t)=\left(\frac{\alpha}{\pi}\right)^{1 / 4} \exp \left\{-\frac{\alpha t^{2}}{2}\right\} \exp \left\{j \sum_{m=1}^{P} \frac{a_{m-1} t^{m}}{m !}\right\},
$$

where $P$ is the order of the PPSs. To compute the LPFT in (2), the polynomial coefficients $\omega_{1}, \omega_{2}, . ., \omega_{M-1}$ are first estimated and then used to form the polynomial phase. The LPFT is obtained by using an STFT procedure whose input is obtained by modulating $s(\tau)$ with $\exp \left\{j \omega t-j \sum_{m=2}^{M} \frac{\omega_{m-1}(\tau-t)^{m}}{m !}\right\}$.

A few important issues, that have direct impacts on the resolution of time-frequency representation and the required computational complexity, have to be addressed. It is always desired to accurately estimate $\omega_{1}$, $\omega_{2}, .$. , and $\omega_{M-1}$ so that a high resolution of the signal representation in the time-frequency domain can be obtained. The polynomial time frequency transform 
(PTFT) $[20,21]$, which is considered as a maximum likelihood estimator, can be used to estimate the polynomial coefficients used in (2). The PTFT is a transform which converts a 1-D input signal into a multi-dimensional output from which the polynomial coefficients are estimated. The maximum number of polynomial coefficients that can be estimated is the same as the order of the PTFT. Figure 1 shows a 2D PTFT of two chirp components. From the location coordinates of the two peaks, the values of $\omega$ and $\omega_{1}$ for the two chirp components can be approximately achieved.

When $M$, the order of the LPFT, is large, one practical problem is that the corresponding PTFT requires a heavy computational load even using the fast algorithms reported in [22,23]. Based on the radix-2 fast algorithm for the PTFT [22] and the fast Fourier transform (FFT) algorithm [24-26] for the STFT computation, the number of complex multiplications needed by the LPFT is approximately

$$
C_{\mathrm{LPFT}}(N) \simeq\left[C_{\mathrm{PTFT}}^{r}(q)+\frac{q}{2} \log _{2} q+0.75 q\right] \frac{N}{q-l^{\prime}}
$$

where $N$ is the length of the input signal, $q$ is the length of the window (or signal segment), and $l, 0 \leq l \leq$ $q-1$, is the length of overlap between signal segments. In (4), $C_{\text {PTFT }}^{r}(q)$ is the number of complex multiplications needed by the $r$ th order PTFT of length $q$, the second term in the square brackets is the number of complex multiplications for an FFT of length $q$, the third term in the square brackets is the number of complex multiplications for windowing and modulation operations, and the factor outside the square brackets is approximately the number of the segments used for the LPFT. It was reported that for the $r$ th order PTFT of length $N$, where $r=2$ or 3 , the required number of complex multiplications are in the order of $N^{r}$ [22]. Because the PTFT requires a heavy computational complexity, especially when $N$ is large, it is important to find various methods to minimize the computational complexity for the parameter estimation by using the PTFT.

Similar to the concept of the STFT which uses Fourier transform to deal with signal segments that contain time-varying frequencies, we can use a smaller number of the polynomial coefficients in the LPFT to approximate the frequency contents of the signal segments. If the second order LPFT is used to process higher-order PPSs, as shown in $[27,28]$ for example, the number of polynomial coefficients to be estimated is reduced, which directly leads to the reduction of the PTFT order, or equivalently the reduction of the required computational complexity. We will discuss the side effects of this order mismatch, i.e., the order of the LPFT is smaller than the order of the PPSs, on the resolution of the signal representation in the time-frequency domain. Other practical issues, which also exist in the STFT, including the effects of window width and the length of overlap between adjacent signal segments will be discussed. The computational complexity can be significantly reduced if

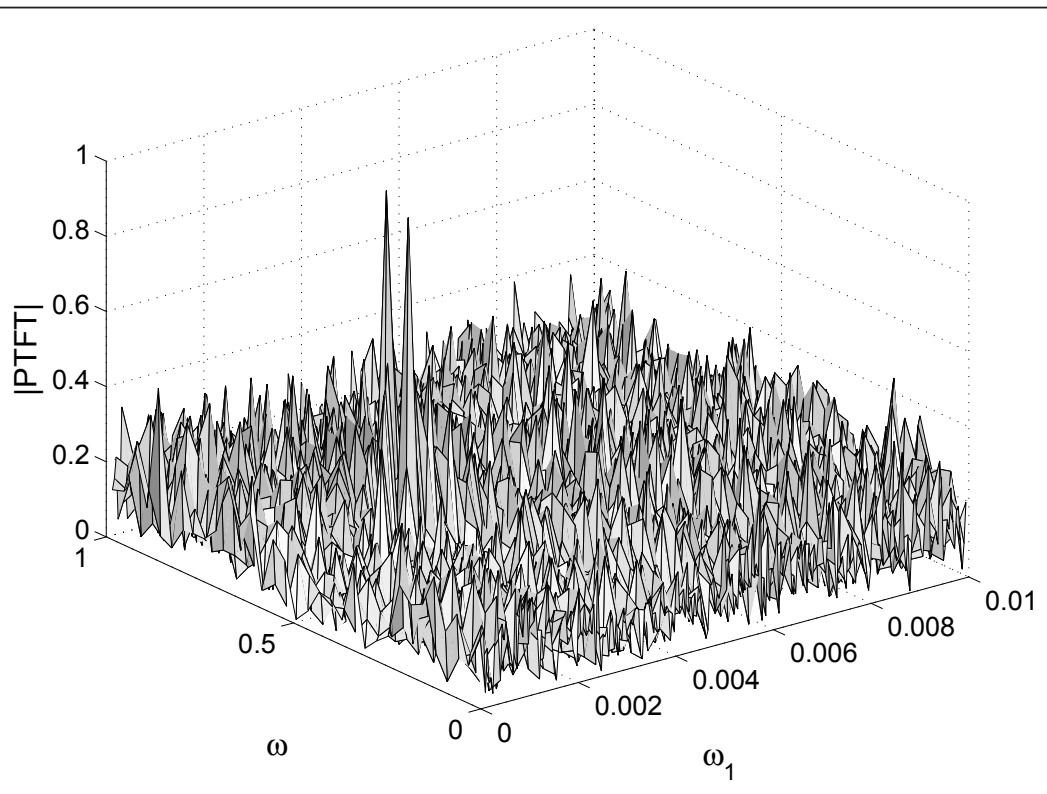

Figure 1 2D PTFT of two chirp components. From the location coordinates of the two peaks, the values of $\omega$ and $\omega_{1}$ for the two chirp components can be approximately achieved. 
the overlap length can be minimized without obviously degrading the resolution of the time-frequency representation.

\subsection{Uncertainty products of the LPFTs}

This subsection first presents the uncertainty principles for the lower order LPFTs, i.e., $M=2,3$ and 4 . Then, a general expression of the uncertainty principle for the $M$ th order LPFT is deduced.

It is assumed that the input signals are PPSs with the same orders of the LPFTs, i.e., $M$ in (2) is equal to $P$ in (3). The corresponding normalized local signal is

$$
\eta_{t}(\tau)=\frac{s(\tau) h(\tau-t) \exp \left\{j \omega t-j \sum_{m=2}^{M} \frac{\omega_{m-1}(\tau-t)^{m}}{m !}\right\}}{\sqrt{\int|s(\tau) h(\tau-t)|^{2} d \tau}},
$$

where

$$
h(t)=\left(\frac{a}{\pi}\right)^{1 / 4} \exp \left\{-\frac{a t^{2}}{2}\right\},
$$

is a Gaussian window used to obtain the signal segments and $a>0$ is the parameter to control the window width

With the above defined input signal and the window function, the normalization factor, i.e., the integral in the denominator of (5), becomes

$$
\int|s(\tau) h(\tau-t)|^{2} d \tau=\left(\frac{a \alpha}{\pi(a+\alpha)}\right)^{1 / 2} \exp \left\{-\frac{a \alpha t^{2}}{a+\alpha}\right\} .
$$

Therefore, the normalized local signal is expressed as

$$
\begin{aligned}
\eta_{t}(\tau)= & \left(\frac{\alpha+a}{\pi}\right)^{1 / 4} \\
& \cdot \exp \left\{-\frac{a^{2} t^{2}}{2(\alpha+a)}\right\} \exp \left\{-\frac{(\alpha+a) \tau^{2}}{2}+a \tau t+j \sum_{m=1}^{p} \frac{a_{m-1} t^{m}}{m !}\right\} \\
& \cdot \exp \left\{j \omega t-j \sum_{m=2}^{M} \frac{\omega_{m-1}(\tau-t)^{m}}{m !}\right\} .
\end{aligned}
$$

For an arbitrary order PPS, it is easy to derive

$$
\left|\eta_{t}(\tau)\right|^{2}=\left(\frac{\alpha+a}{\pi}\right)^{1 / 2} \exp \left\{-(\alpha+a)\left(\tau-\frac{a t}{\alpha+a}\right)^{2}\right\},
$$

which is independent of the order of the PPS. With (32) in Appendix, the mean time, $\langle\tau\rangle_{t}$, of the normalized local signal becomes

$$
\langle\tau\rangle_{t}=\frac{a t}{a+\alpha} .
$$

Similarly, we also obtain

$$
\left\langle\tau^{2}\right\rangle_{t}=\frac{1}{2(a+\alpha)}+\frac{a^{2} t^{2}}{(a+\alpha)^{2}} .
$$

Therefore the time duration of the normalized local signal, which is defined in (33) in Appendix, is expressed as

$$
T_{t}^{2}=\frac{1}{2(a+\alpha)}
$$

Since both $\langle\tau\rangle_{t}$ and $T_{t}^{2}$ are related to $\left|\eta_{t}(\tau)\right|$ instead of $\eta_{t}(\tau)$, they are independent of the order of the input signal. It means that the signal duration in the time domain is determined by the signal parameter $\alpha$ and window parameter $a$ only.

Let us now compute the mean frequency $\langle\omega\rangle_{t}$ and the bandwidth $B_{t}^{2}$ for the LPFTs of various orders. For the second order LPFT, the local signal is

$$
s_{t}(\tau)=s(\tau) h(\tau-t) \exp \left\{j \omega t-j \frac{\omega_{1}(\tau-t)^{2}}{2}\right\},
$$

where $s(t)$, the input of the second order LPFT, is the second order PPS or the chirp signal, obtained by setting $P=2$ in (3), and $\omega_{1}$ is estimated by using the second order PTFT. In this case, the Fourier transform of the local signal, $s_{t}(\tau)$, becomes the second order LPFT of $s(t)$.

The normalized local signal for $P=2$ in (8) at the time $t$ becomes

$$
\begin{aligned}
\eta_{t}(\tau)= & \frac{s(\tau) h(\tau-t) \exp \left\{j \omega t-j \frac{\omega_{1}(\tau-t)^{2}}{2}\right\}}{\sqrt{\int|s(\tau) h(\tau-t)|^{2} d \tau}} \\
= & \left(\frac{\alpha+a}{\pi}\right)^{1 / 4} \exp \left\{-\frac{(\alpha+a) \tau^{2}}{2}+a \tau t+j a_{0} \tau+\frac{j a_{1} \tau^{2}}{2}-\frac{a^{2} t^{2}}{2(\alpha+a)}\right\} \\
& \cdot \exp \left\{j \omega t-\frac{j \omega_{1}(\tau-t)^{2}}{2}\right\} .
\end{aligned}
$$

With the definition in (35), we have

$$
\langle\omega\rangle_{t}=a_{0}+\omega_{1} t+\frac{a t\left(a_{1}-\omega_{1}\right)}{a+\alpha}=a_{0}+\omega_{1} t+\left(a_{1}-\omega_{1}\right)\langle\tau\rangle_{t},
$$

in which the first term is related to the signal parameter $a_{0}$, the second term is related to $\omega_{1}$, and the last term is a product of the deviation of $\omega_{1}$ from $a_{1}$ and the mean time, $\langle\tau\rangle_{t}$.

Similarly, by using (37) in Appendix, we have

$$
\left\langle\omega^{2}\right\rangle_{t}=\frac{a+\alpha}{2}+\langle\omega\rangle_{t}^{2}+\frac{\left(a_{1}-\omega_{1}\right)^{2}}{2(a+\alpha)},
$$

which shows that the frequency variance is determined by the parameters of the signal and the window in the first term, the square of mean frequency in the second term, and the scaled square of the estimation error of the polynomial parameter $\omega_{1}$ in the last term. With the above derivation results, the bandwidth in the domain of the second order LPFT becomes 


$$
B_{t}^{2}=\left\langle\omega^{2}\right\rangle_{t}-\langle\omega\rangle_{t}^{2}=\frac{a+\alpha}{2}+\frac{\left(a_{1}-\omega_{1}\right)^{2}}{2(a+\alpha)} .
$$

Therefore

$$
B_{t}^{2} T_{t}^{2}=\frac{1}{4}+\frac{\left(a_{1}-\omega_{1}\right)^{2}}{4(a+\alpha)^{2}}
$$

which is related to the parameters of the signal and the window, and the errors of estimating the polynomial parameters $\omega_{1}$.

Let us next consider the uncertainty principle of the third order LPFT. The local signal is

$$
s_{t}(\tau)=s(\tau) h(\tau-t) \exp \left\{j \omega t-j \frac{\omega_{1}}{2}(\tau-t)^{2}-j \frac{\omega_{2}}{6}(\tau-t)^{3}\right\},
$$

where $s(t)$, the input of the third order LPFT, is the third order PPS obtained by setting $P=3$ in (3).

In this case, the local normalized signal becomes

$$
\begin{aligned}
\eta_{t}(\tau)= & \left(\frac{\alpha+a}{\pi}\right)^{1 / 4} \\
& \cdot \exp \left\{-\frac{(\alpha+a) \tau^{2}}{2}+a \tau t+j a_{0} \tau+\frac{j a_{1} \tau^{2}}{2}+\frac{j a_{2} \tau^{3}}{6}-\frac{a^{2} t^{2}}{2(\alpha+a)}\right\} \\
& \cdot \exp \left\{j \omega t-\frac{j \omega_{1}(\tau-t)^{2}}{2}-\frac{j \omega_{2}(\tau-t)^{3}}{6}\right\} .
\end{aligned}
$$

With the same procedure, the mean frequency, defined in (35) in Appendix, is expressed as

$$
\langle\omega\rangle_{t}=a_{0}+\omega_{1} t-\frac{\omega_{2} t^{2}}{2}+\left(a_{1}-\omega_{1}+\omega_{2} t\right)\langle\tau\rangle_{t}+\frac{1}{2}\left(a_{2}-\omega_{2}\right)\left\langle\tau^{2}\right\rangle_{t},
$$

and $\left\langle\omega^{2}\right\rangle_{t}$, defined in (37) in Appendix, becomes

$$
\begin{aligned}
\left\langle\omega^{2}\right\rangle_{t}= & \frac{a+\alpha}{2}+\langle\omega\rangle_{t}^{2}+\frac{1}{2(a+\alpha)}\left[\left(a_{1}-\omega_{1}+\omega_{2} t\right)+\left(a_{2}-\omega_{2}\right)\langle\tau\rangle_{t}\right]^{2} \\
& +\frac{\left(a_{2}-\omega_{2}\right)^{2}}{8(a+\alpha)^{2}}
\end{aligned}
$$

Thus, the bandwidth becomes

$$
\begin{aligned}
B_{t}^{2} & =\left\langle\omega^{2}\right\rangle_{t}-\langle\omega\rangle_{t}^{2} \\
& =\frac{a+\alpha}{2}+\frac{\left[\left(a_{1}-\omega_{1}+\omega_{2} t\right)+\left(a_{2}-\omega_{2}\right)\langle\tau\rangle_{t}\right]^{2}}{2(a+\alpha)}+\frac{\left(a_{2}-\omega_{2}\right)^{2}}{8(a+\alpha)^{2}} .
\end{aligned}
$$

Therefore

$$
B_{t}^{2} T_{t}^{2}=\frac{1}{4}+\frac{\left[\left(a_{1}-\omega_{1}+\omega_{2} t\right)+\left(a_{2}-\omega_{2}\right)\langle\tau\rangle_{t}\right]^{2}}{4(a+\alpha)^{2}}+\frac{\left(a_{2}-\omega_{2}\right)^{2}}{16(a+\alpha)^{3}} .
$$

It is observed that the uncertainty product, $B_{t}^{2} T_{t}^{2}$, is affected by the errors of estimating the polynomial parameters $\omega_{1}$ and $\omega_{2}$ in addition to the parameters of the signal and the window function.

By setting $P=4$ in (3) and using the fourth order LPFT, we can similarly obtain the corresponding mean frequency $\langle\omega\rangle_{t}$ and the bandwidth $B_{t}^{2}$, which are listed in Table 1 respectively. From the expressions in the table, we can observe the rules of $\langle\omega\rangle_{t}$ and $B_{t}^{2}$ as $M$, the order of the LPFT, is increased. The mean frequency and the bandwidth for the Mth-order LPFT are

$$
\begin{aligned}
\langle\omega\rangle_{t} & =a_{0}+\sum_{m=2}^{M} \frac{(-1)^{m} \omega_{m-1} t^{m-1}}{(m-1) !} \\
& +\sum_{n=1}^{M-1} \frac{1}{n !}\left(a_{n}+\sum_{m=n+1}^{M} \frac{(-1)^{m-n} \omega_{m-1} t^{m-n-1}}{(m-n-1) !}\right)\left\langle\tau^{n}\right\rangle_{t} \\
B_{t}^{2} & =\frac{1}{4 T_{t}^{2}}+\sum_{l=1}^{M-1} \frac{T_{t}^{2 l}}{l !} \\
& \cdot\left[\sum_{n=l}^{M-1} \frac{1}{(n-l) !}\left(a_{n}+\sum_{m=n+1}^{M} \frac{(-1)^{m-n} \omega_{m-1} t^{m-n-1}}{(m-n-1) !}\right)\left\langle\tau^{n-l}\right\rangle_{t}\right]^{2} .
\end{aligned}
$$

\begin{tabular}{|c|c|}
\hline$M$ & \\
\hline & $\langle\omega\rangle_{t}$ \\
\hline 2 & $a_{0}+\omega_{1} t+\left(a_{1}-\omega_{1}\right)\langle\tau\rangle_{t}$ \\
\hline 3 & $a_{0}+\omega_{1} t-\frac{\omega_{2} t^{2}}{2}+\left(a_{1}-\omega_{1}+\omega_{2} t\right)\langle\tau\rangle_{t}+\frac{1}{2}\left(a_{2}-\omega_{2}\right)\left\langle\tau^{2}\right\rangle_{t}$ \\
\hline & $a_{0}+\omega_{1} t-\frac{\omega_{2}}{2} t^{2}+\frac{\omega_{3}}{6} t^{3}+\left(a_{1}-\omega_{1}+\omega_{2} t-\frac{\omega_{3}}{2} t^{2}\langle\tau\rangle_{t}\right.$ \\
\hline 4 & $\begin{array}{c}+\frac{1}{2}\left(a_{2}-\omega_{2}+\omega_{3} t\right)\left\langle\tau^{2}\right\rangle_{t}+\frac{1}{6}\left(a_{3}-\omega_{3}\right)\left\langle\tau^{3}\right\rangle_{t} \\
B_{t}^{2}\end{array}$ \\
\hline 2 & $\frac{a+\alpha}{2}+\frac{1}{2(a+\alpha)}\left(a_{1}+\omega_{1}\right)^{2}$ \\
\hline 3 & $\frac{a+\alpha}{2}+\frac{1}{2(a+\alpha)}\left[\left(a_{1}-\omega_{1}+\omega_{2} t\right)+\left(a_{2}-\omega_{2}\right)\langle\tau\rangle_{t}\right]^{2}+\frac{1}{8(a+\alpha)^{2}}\left(a_{2}-\omega_{2}\right)^{2}$ \\
\hline & $\frac{a+\alpha}{2}+\frac{1}{2(a+\alpha)}\left[\left(a_{1}-\omega_{1}+\omega_{2} t-\frac{\omega_{3}}{2} t^{2}\right)+\left(a_{2}-\omega_{2}+\omega_{3} t\right)\langle\tau\rangle_{t}\right.$ \\
\hline 4 & $\begin{aligned}\left.+\frac{1}{2}\left(a_{3}-\omega_{3}\right)\left\langle\tau^{2}\right\rangle_{t}\right]^{2} & \left.+\frac{1}{8(a+\alpha)^{2}}\left(a_{2}-\omega_{2}+\omega_{3} t\right)+\left(a_{3}-\omega_{3}\right)\langle\tau\rangle_{t}\right]^{2} \\
& +\frac{1}{48(a+\alpha)^{3}}\left(a_{3}-\omega_{3}\right)^{2}\end{aligned}$ \\
\hline
\end{tabular}

The lengthy mathematical derivation of the above two equations is omitted due to the space limit. It is observed that both $\langle\omega\rangle_{t}$ and $B_{t}^{2}$ are related to the moments $\left\langle\tau^{n}\right\rangle_{t}$. The expressions of the moments $\left\langle\tau^{n}\right\rangle_{t}$, for $n=1,2, . ., 6$, which can be calculated from (34), are listed in Table 2.

In general the uncertainty product of an arbitrary order LPFT is related to the parameters of the signal and the window function, as well as the errors of estimating the polynomial coefficients. When the polynomial coefficients are estimated correctly for the $M$ th order LPFT, that is, $\omega_{1}=\frac{d \Omega(t)}{d t}, . ., \omega_{M-1}=\frac{d^{M-1} \Omega(t)}{d t^{M-1}}$, the mean frequency becomes

Table 1 Expressions of $\langle\omega\rangle_{t}$ and $B_{t}^{2}$ for LPFTs of order 2, 3 and 4 
Table 2 Expressions of $\left\langle\tau^{n}\right\rangle_{t}$ for $n=1,2,34,5$ and 6

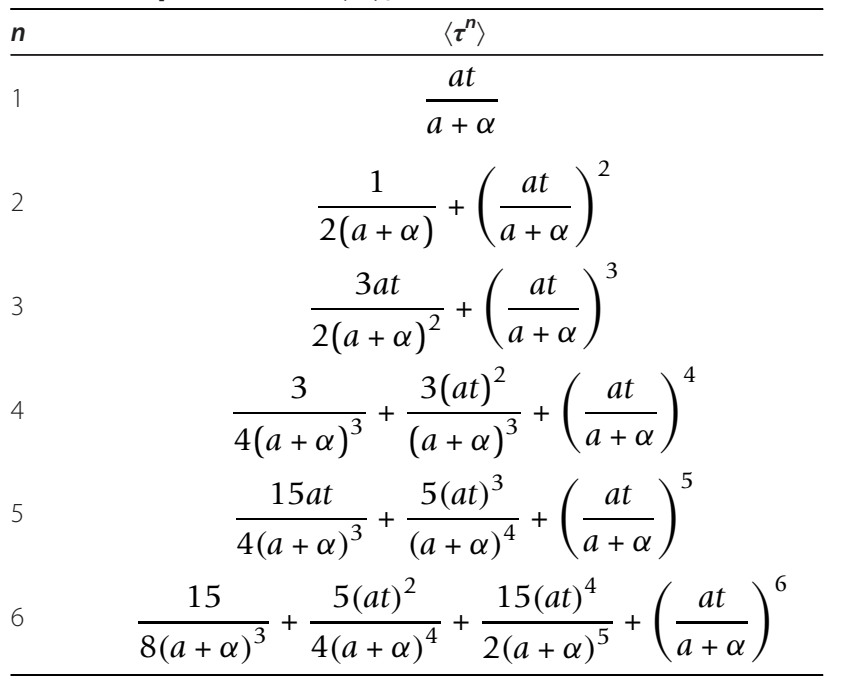

$$
\langle\omega\rangle_{t}=a_{0}+a_{1} t+\frac{a_{2}}{2} t^{2}+\cdots+\frac{a_{M-1}}{(M-1) !} t^{M-1}=\sum_{m=1}^{M} \frac{a_{m-1} t^{m-1}}{(m-1) !}
$$

which is exactly the instantaneous frequency of the Mth order PPS.

Without giving further details, the bandwidth in the domain of the $M$ th order LPFT is derived as $B_{t}^{2}=\frac{a+\alpha}{2}$ when the polynomial parameters are estimated correctly, which leads to the uncertainty product to be

$$
B_{t}^{2} T_{t}^{2}=\frac{1}{4}
$$

Based on the above derived results, we have the following conclusions. When using the Gaussian window to segment the signal, the signal duration of the PPS is only related to the signal parameter $\alpha$, the window parameter $a$ and is independent of the order. For $M>1$, the bandwidth $B_{t}^{2}$ is related to the parameters of the signal and the window function, as well as the errors of estimating the polynomial parameters $\omega_{1}, \omega_{2} \ldots$, and $\omega_{M-1}$. When the polynomial coefficients are estimated accurately, the uncertainty product of the $M$ th order LPFT, with the same order PPS as the input, is independent of time or frequency, and becomes a constant. When there are errors of estimating the polynomial coefficients, the bandwidth together with the uncertainty product, are polynomial functions of these errors, which directly affect the signal representation in the time-frequency domain.

It should be noted that in this article the uncertainty principle of the LPFT is derived based on the PPSs. For other kind of time-varying signals such as sinusoidal FM signals or parabolic FM signals, we can use the window function to segment the signals to ensure that each segment within the window can be assumed to be the PPS. As long as the signal segments can be modeled as PPSs, the above presented results are still valid. In this way, the derived uncertainty principle of the LPFT can also be extended to general time-varying signals.

\section{Discussions}

The main objective on studying various issues of the LPFT is to achieve the desired signal resolution in the time-frequency domain with minimum computational costs. This section considers a few important issues, in terms of the uncertainty principles of the LPFTs, that have direct impacts on signal resolution and the required computational complexity.

\subsection{Window width effects}

The uncertainty relations derived in the previous section show that there exists a trade-off between the resolutions in the time and frequency domains. With the assumption that the polynomial coefficients used in the LPFT are accurately estimated, the signal duration $T_{t}^{2}$ is inversely proportional to the sum of window width $a$ and the signal parameter $\alpha$, while the signal bandwidth $B_{t}^{2}$ is directly proportional to this sum, regardless of the order of the PPS and the LPFT. With different widths of the Gaussian window, Figure 2 shows the signal concentrations in the time-frequency domain achieved by using the second order LPFT to process the PPS which contains chirp components.

It is seen that, as a decreases or the window width increases, the chirp components become more concentrated in the frequency domain, or equivalently, the resolution of the signal representation in the frequency direction is increased. In contrast, the resolution in the time direction decreases. This observation is consistent with the signal duration $T_{t}^{2}$ and the bandwidth $B_{t}^{2}$ derived in the last Section. For example, decreasing the window parameter $a$ leads to the increase of the signal duration $T_{t}^{2}$, or equivalently, the increase of signal spread in time. At the same time, the bandwidth $B_{t}^{2}$ decreases as $a$ decreases so that the signal spread in frequency is reduced, that is, the resolution of the signal representation in the frequency direction is increased. Because it is impossible to increase the resolution in both domains at the same time, a compromise has to be made to balance the requirements of the signal representation in the time-frequency domain. When $a=2.5$, for example, Figure 2c provides acceptable resolutions for both time and frequency domains, that is, the resolution in the time domain and the frequency domain is high enough to help us clearly separating the closely located chirp components. 


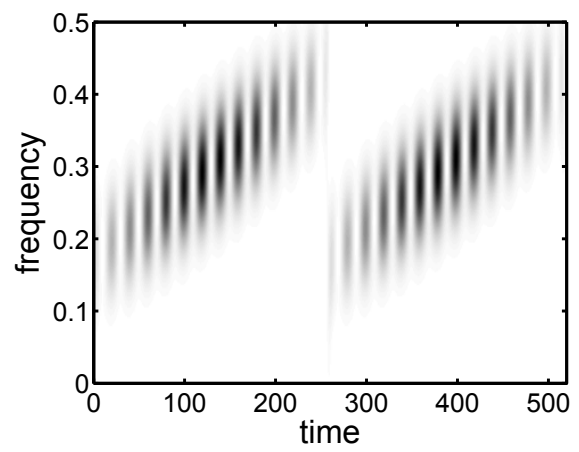

(a) $a=25$

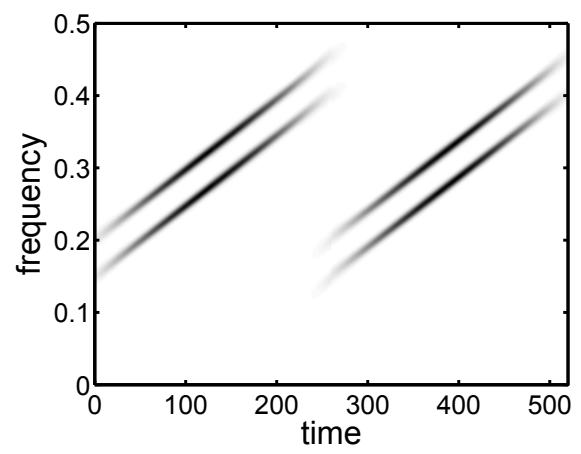

(c) $a=2.5$

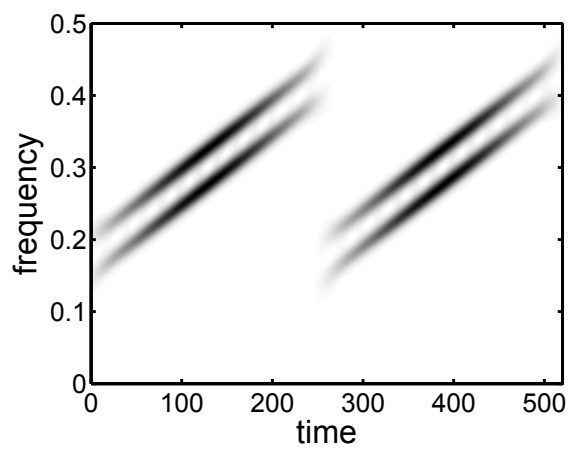

(b) $a=5$

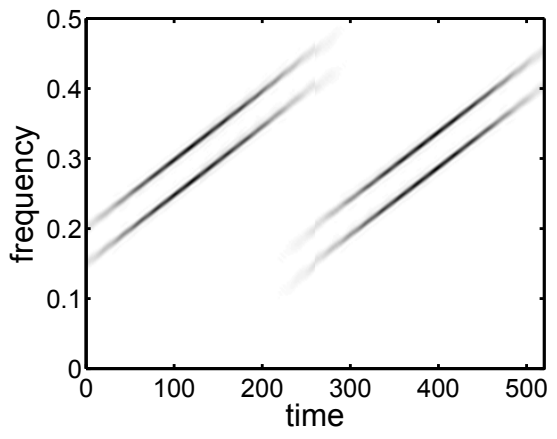

(d) $a=0.25$

Figure 2 The second order LPFTs of a second order PPS. The window length is $1 / 4$ of the signal length.

\subsection{Order mismatch effects}

As discussed in the Section 1, it is desired to use lower order LPFTs to deal with higher-order PPSs to significantly reduce the required computational complexity. However, this mismatch between the orders of the LPFT and the PPSs will affect the performance of the signal representation in the time-frequency domain. It is necessary to find out how the signal representation in the time-frequency domain is affected and if possible, how the mismatch effects are to be minimized. Because it is difficult to draw a general conclusion for all possible cases, let us consider the use of the second order LPFT to process the third order PPSs.

By setting $\omega_{2}=0$ in (17) and (19), the mean frequency and the bandwidth become

$$
\begin{aligned}
\langle\omega\rangle_{t} & =a_{0}+\omega_{1} t+\left(a_{1}-\omega_{1}\right)\langle\tau\rangle_{t}+\frac{1}{2} a_{2}\left\langle\tau^{2}\right\rangle_{t} \\
& =a_{0}+\omega_{1} t+\frac{a t\left(a_{1}-\omega_{1}\right)}{a+\alpha}+\frac{a_{2}\left(2 a^{2} t^{2}+a+\alpha\right)}{4(a+\alpha)^{2}} \\
B_{t}^{2} & =\frac{a+\alpha}{2}+\frac{1}{2(a+\alpha)}\left[\left(a_{1}-\omega_{1}\right)+\frac{a_{2} a t}{a+\alpha}\right]^{2}+\frac{a_{2}^{2}}{8(a+\alpha)^{2}} .
\end{aligned}
$$

When the parameter $\omega_{1}$ is estimated correctly, i.e., $\omega_{1}$ $=a_{1}+a_{2} t$, we have

$$
\begin{aligned}
& \langle\omega\rangle_{t}=a_{0}+a_{1} t+\frac{a_{2} t^{2}}{2}+\frac{a^{2} a_{2} t^{2}}{2(a+\alpha)^{2}}+\frac{a_{2}}{4(a+\alpha)} \\
& B_{t}^{2}=\frac{a+\alpha}{2}+\frac{\left(a_{2} a t\right)^{2}}{2(a+\alpha)^{3}}+\frac{a_{2}^{2}}{8(a+\alpha)^{2}} .
\end{aligned}
$$

Therefore, the uncertainty product becomes

$$
B_{t}^{2} T_{t}^{2}=\frac{1}{4}+\frac{1}{4(a+\alpha)^{2}}\left(\frac{a_{2} a t}{a+\alpha}\right)^{2}+\frac{a_{2}^{2}}{16(a+\alpha)^{3}} .
$$

In (23) or (24), the last term is the effects of the order mismatch, which are directly proportional to the polynomial coefficient, $a_{2}$. Therefore, the order mismatch may have a small effect when $a_{2}$ is small enough, which is generally true in many practical applications. Another way to reduce the effect of the order mismatch is to minimize the signal duration $T_{t}^{2}$ by decreasing the window width or increasing the window parameter $a$. For example, when $a \rightarrow \infty,\langle\omega\rangle_{t}$ in (23) is approaching to $a_{0}+a_{1} t+\frac{a_{2}}{2} t^{2}$, which is the instantaneous frequency of the third order PPS. Meanwhile $B_{t}^{2}$ in (24) is approaching to $\frac{a+\alpha}{2}$. It means that, when the third order PPS is processed with the second 
order LPFT, $\langle\omega\rangle_{t}$ and $B_{t}^{2}$ of the second order LPFT approach to those of the third order LPFT as the window width is decreased. The uncertainty product in (25) also approaches to the minimum.

When the signal parameter $\alpha$ approaches to zero, the third order PPS with a Gaussian amplitude becomes a third order PPS with a constant amplitude. Under this situation, the mean frequency and bandwidth become

$$
\begin{aligned}
\langle\omega\rangle_{t} & =a_{0}+a_{1} t+\frac{a_{2} t^{2}}{2}+\frac{a_{2}}{4 a} \\
B_{t}^{2} & =\frac{a}{2}+\frac{a_{2}^{2}}{8 a^{2}}
\end{aligned}
$$

and the uncertainty product is

$$
B_{t}^{2} T_{t}^{2}=\frac{1}{4}+\frac{a_{2}^{2}}{16 a^{3}} \quad \text { as } \quad \alpha \rightarrow 0 \text {, }
$$

which increases with the increase of the polynomial coefficient $a_{2}$ and with the decrease of the window parameter $a$. Similarly, as $a$ goes to infinity, $\langle\omega\rangle_{t}$ approaches to the instantaneous frequency, and $B_{t}^{2} T_{t}^{2}$ approaches to $\frac{1}{4}$ which is the minimum of the uncertainty product.

From the above discussion, it is seen that increasing the window parameter $a$ always helps to achieve a better estimation of the instantaneous frequency $\langle\omega\rangle_{t}$. It should be noted that, however, as $a$ goes to infinity, that is as the window becomes narrower in the time domain, the signal bandwidth $B_{t}^{2}$, or the spread in frequency in (24) also approaches to infinity, which decreases the resolution in frequency. Therefore, the window width for practical applications should be properly selected to achieve a compromise between the resolutions in both time and frequency. An adaptive procedure for window length selection has been provided in [28]. Similarly, an automated procedure for window width selection is possible and will be discussed in our future study.

\subsection{Effects of overlap lengths}

It was shown in [28] that when the second order LPFT is used to process the chirp signals, the length of overlap between the adjacent signal segments can be significantly reduced without obviously degrading the signal resolution in the time-frequency domain, which allows us to further minimize the required computational complexity. For example, the required number of complex multiplications in (4) is reduced as $l$ increases. For the third order PPS,

$$
\begin{aligned}
s(t) & =\left(\frac{\alpha}{\pi}\right)^{1 / 4} e^{-\alpha t^{2} / 2}\left[e^{j\left(0.4 t+1.15 \times 10^{-3} t^{2}+1.48 \times 10^{-6} t^{3}\right)}\right. \\
& \left.+e^{j\left(0.45 t+1.15 \times 10^{-3} t^{2}+1.48 \times 10^{-6} t^{3}\right)}\right]
\end{aligned}
$$

with $\alpha=10^{-4}$, Figure 3a shows the signal representation obtained by the third order LPFT without any overlap. Compared to the performance with the maximum overlap, i.e., $l=q-1$, as shown in Figure $3 \mathrm{~b}$, no obvious degradation in signal resolution is observed compared to Figure 3a. Because the total computational complexity is directly proportional to the number of signal segments being processed, the LPFT computation without overlap between signal segments makes significant savings on the computational complexity compared to that using the overlap between signal segments.

Let us next consider the effects of using lower order LPFT with different overlap lengths. Figure 4a shows the representation of the third order PPS obtained by using the second order LPFT without any overlap. Obvious unsmoothness is observed at the junctions of two adjacent segments where the values of the estimated chirp rates have sudden changes. This order mismatch effect can be minimized by increasing the length of overlap between the adjacent segments. As shown in Figure $4 \mathrm{~b}$, the second order LPFT with the maximum overlap achieves a representation that is comparable to that obtained by using the third order LPFT in Figure 3b.

To process higher-order PPSs with the second order LPFT, another method for improvement on signal representation is to reduce the window length to ensure that each segment can be approximately assumed as a chirp component. Compared with Figure 4a, c shows the improvement on the smoothness of signal representation, which is achieved by reducing the window length from $1 / 4$ to $1 / 8$ of the signal length. It should be noted that, compared with the third order LPFT with the small window length in Figure $4 \mathrm{~d}$, the second order LPFT with the same window length in Figure 4c can achieve comparable result and with less computational complexity. It is worth mentioning that for any order LPFTs, the frequency resolution will become degraded as the window length is reduced, as shown in Figure 4c, d. For other higher-order PPSs, the second order LPFT can also be used, as long as the window length is small enough to ensure that each segment within the window can be approximately assumed as the chirp signal.

\subsection{Resolution comparisons}

Let us now consider the performances achieved by the STFT and the second order LPFT of a chirp signal obtained from (3) with $P=2$. Using the STFT, the corresponding mean frequency and bandwidth are [29]

$$
\langle\omega\rangle_{t}=a_{0}+\frac{\alpha a_{1} t}{a+\alpha}, \quad B_{t}^{2}=\frac{a+\alpha}{2}+\frac{a_{1}^{2}}{2(a+\alpha)} .
$$




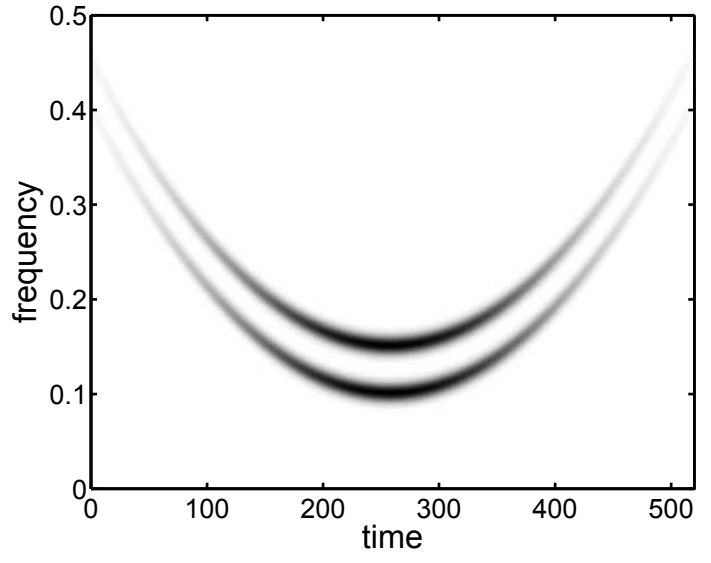

(a)

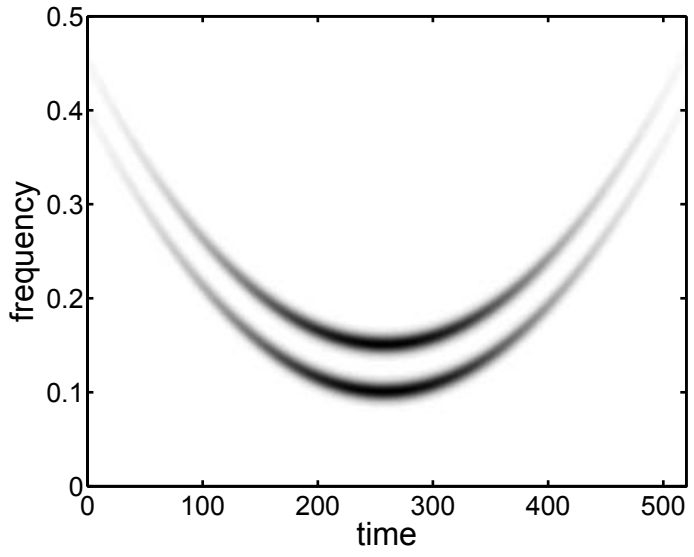

(b)

Figure 3 The third order LPFTs of a third order PPS with $a=2.5$ (the window length is $\mathbf{1 / 4}$ of the signal length). (a) The LPFT without overlap between adjacent segments, and (b) The LPFT with the maximum overlap.

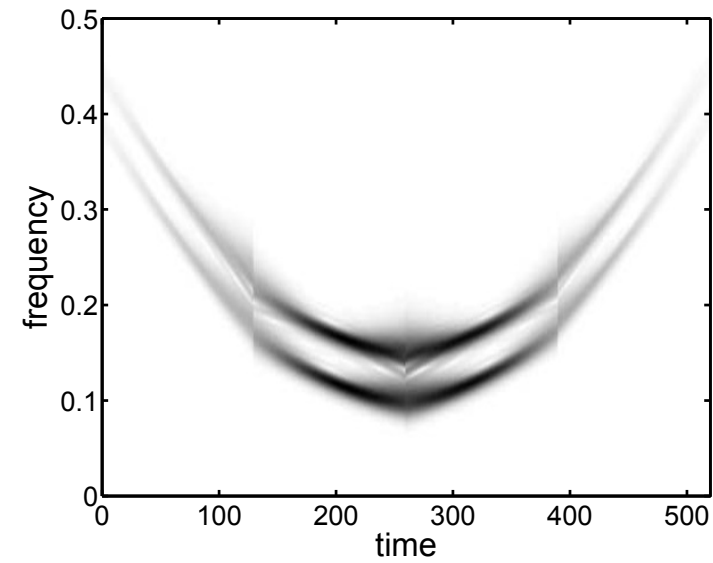

(a)

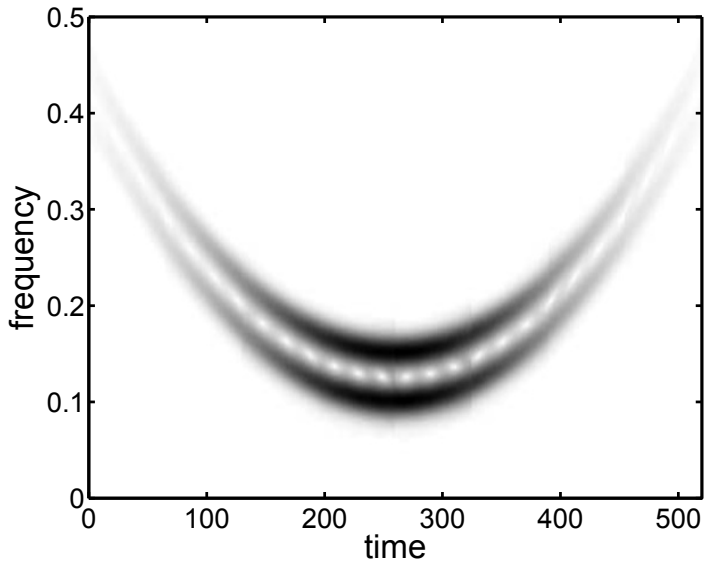

(c)

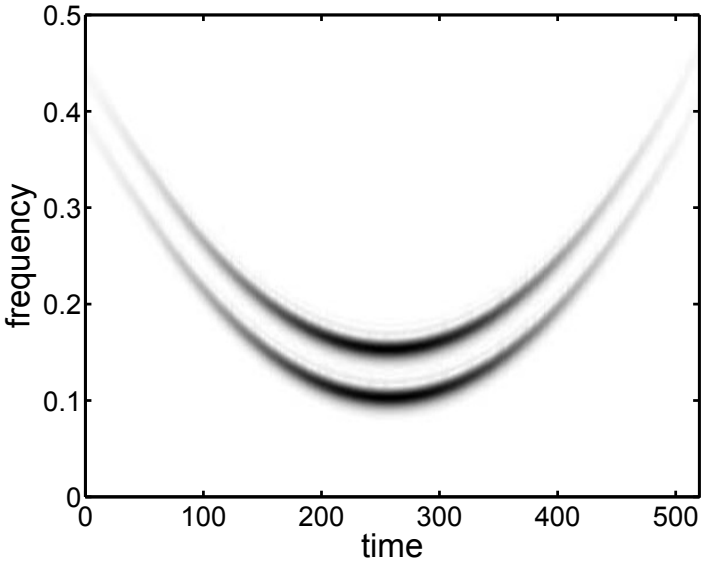

(b)

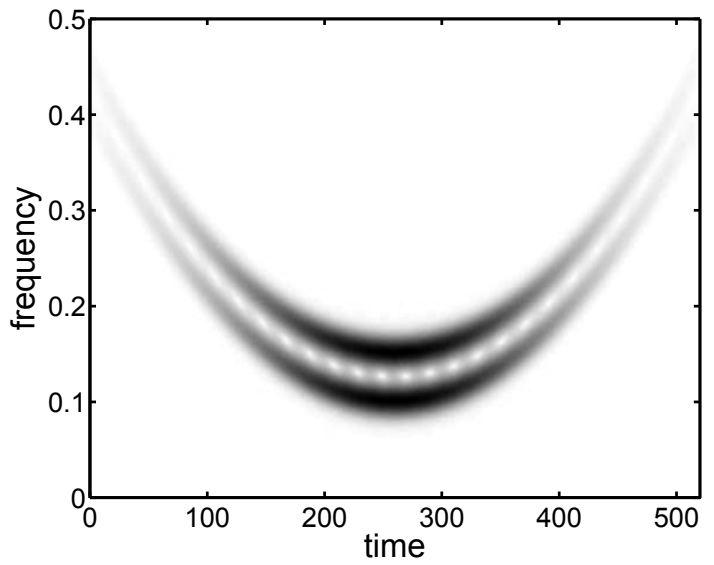

(d)

Figure 4 The LPFTs of the third order PPS with $\boldsymbol{a}=\mathbf{2 . 5}$. (a) The second order LPFT without overlap, (b) the second order LPFT with the maximum overlap, (c) the second order LPFT without overlap and with a shorter window, and (d) the third order LPFT without overlap. The window length for (a) and (b) is $1 / 4$ of signal length and that for $(\mathbf{c})$ and (d) is $1 / 8$ of signal length. 
The mean time $\langle\tau\rangle_{t}$ and the duration $T_{t}^{2}$ of the STFT are the same as those of the LPFT. Therefore the uncertainty product of the STFT is

$$
B_{t}^{2} T_{t}^{2}=\frac{1}{4}+\frac{a_{1}^{2}}{4(a+\alpha)^{2}} .
$$

When $a_{1}=0$, the input signal becomes one whose frequencies do not change with time and the STFT achieves the lowest bound of the uncertainty product $B_{t}^{2} T_{t}^{2}=\frac{1}{4}$. Therefore, the STFT can provide good resolution only for signals with constant frequencies. For the chirp signals, however, $B_{t}^{2}$ in (29) has a non-zero second term. Therefore, the uncertainty product $B_{t}^{2} T_{t}^{2}$ of the STFT of time-varying signals must be larger than $\frac{1}{4}$. For the same input signal, if the polynomial coefficient, $\omega_{1}$, of the LPFT is correctly estimated, we achieve the lowest bound of the uncertainty product $B_{t}^{2} T_{t}^{2}=\frac{1}{4}$, as seen in (22). Therefore the second order LPFT achieves a more concentrated distribution in the time-frequency domain than the STFT.

Another important issue on the LPFT is about the estimation errors on $\omega_{1}$. Although the PTFT is the maximum likelihood estimator, it is still possible to have some estimation errors, especially when the signals are corrupted by heavy noises. Because the second term of (15) is proportional to $\left(a_{1}-\omega_{1}\right)^{2}$, the uncertain product for the second order LPFT of chirp signals is $B_{t}^{2} T_{t}^{2}>\frac{1}{4}$ if $\omega_{1}$ is not correctly estimated. Comparing (15) and (29) reveals that as long as $\left|a_{1}-\omega_{1}\right|<\left|a_{1}\right|$, i.e., the estimation error of $\omega_{1}$ is smaller than $\left|a_{1}\right|$, the LPFT of the chirp signal achieves a smaller bandwidth than the STFT. Therefore, the second order LPFT can still achieve a more concentrated distribution in the timefrequency domain than the STFT. Furthermore, the resolution performance of the STFT cannot be further improved because $a_{1}$ in (29) or (30) is the signal parameter. In comparison, improvement on the resolution performance of the LPFT can be achieved by minimizing the error of parameter estimation. It should be noted that if the estimation error of $\omega_{1}$ is larger than | $a_{1} \mid$, the resolution of the LPFT will become worse than that of the STFT although such a case rarely happens when the PTFT is used to estimate the polynomial coefficients.

Besides the STFT and LPFT, which are linear timefrequency transforms, another bilinear time-frequency transform, known as the WVD, has been often used in many applications [29]. The WVD of the chirp signal is

$$
\mathrm{WVD}(t, \omega)=\frac{1}{\pi} \exp \left\{-\alpha t^{2}-\frac{\left(\omega-a_{1} t-a_{0}\right)^{2}}{\alpha}\right\} .
$$

When is small, the WVD is concentrated on $\omega=a_{0}+$ $a_{1} t$. The associated mean frequency and bandwidth are [29]

$$
\langle\omega\rangle_{t}=a_{0}+a_{1} t \quad \text { and } \quad B_{t}^{2}=\frac{\alpha}{2} .
$$

It means that the WVD can provide a better frequency resolution for chirp signals as far as $\alpha$ is small enough. However, the WVD suffers from the cross terms for multiple-component signals. It is difficult to remove the cross terms without sacrificing the resolution of the signal representation, particularly for nonlinear multiple-component chirp signals [29].

In order to clearly show the frequency resolution of the signal, Figure 5 compares signal concentration obtained from the STFT, LPFT and WVD at a particular time instant. Resolution usually means whether we can separate two closely located components in the time and/or frequency domains, while concentration describes how the transforms concentrate along the signal's instantaneous frequency. Resolution and concentration are closely related and high concentration generally contributes to high resolution. For signals with constant frequency components, the STFT can provide the good frequency resolution, as shown in Figure 5. For chirp signals, with the same window length and window parameter $a$, however, the LPFT achieves a better frequency resolution as seen in Figure 5b, c. Although the WVD also has a better frequency resolution, the cross terms between the two chirp components, as shown in Figure $5 \mathrm{~d}$, can be easily mistaken as a valid frequency component. Therefore, the second order LPFT is the best choice for dealing with chirp signals since it is free from the cross terms and also achieves a better resolution than the STFT.

\section{Conclusion}

In this article, the LPFT is shown to be limited by the uncertainty principle, and the un-certainty relations of various order LPFTs are derived to show the trade-off between the resolutions of signal representation in the time and frequency domains and the required computational complexity. The uncertainty product of an arbitrary order is determined by the signal parameters, the window function and the errors of estimating the polynomial coefficients. When Gaussian window function is employed to segment the signals, the uncertainty products of the LPFT are time independent under the 
(a)

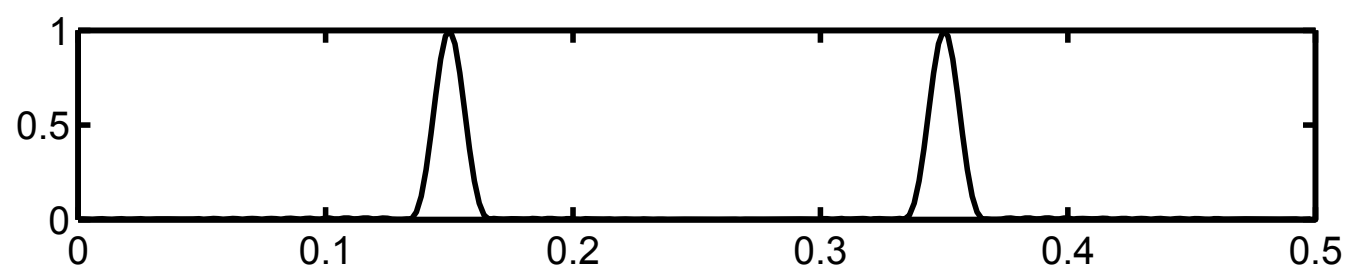

(b)

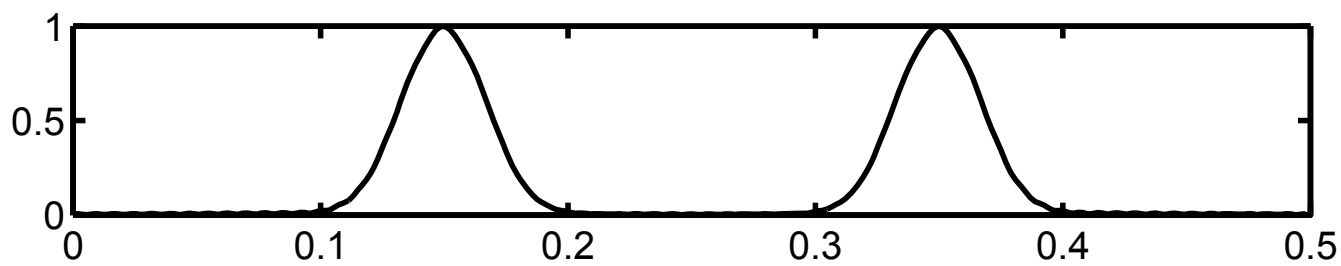

(c)

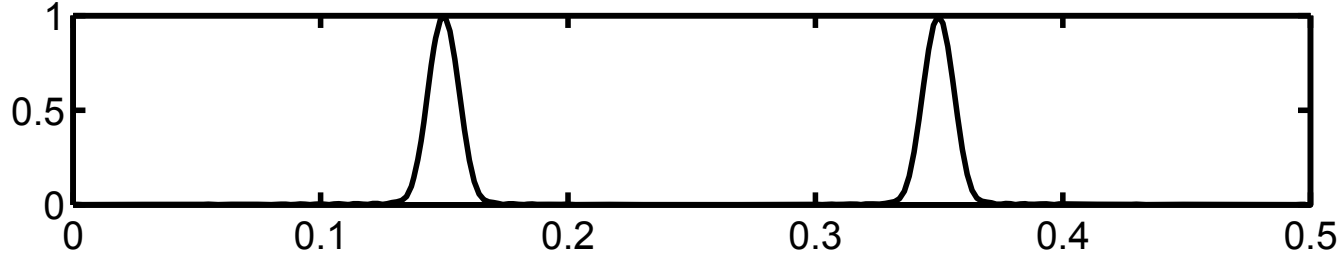

(d)

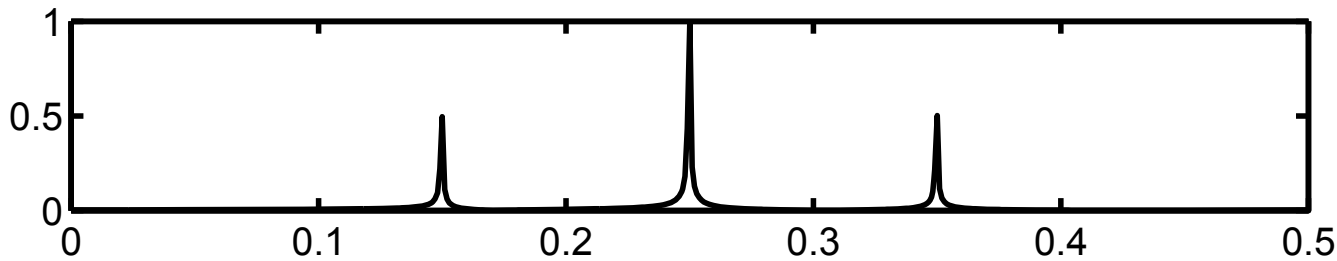

Figure 5 The signal concentration comparisons of different time-frequency rep-resentations for a two-component signal. (a) The STFT of a signal with constant frequencies; (b)-(d) The concentrations of chirp signals using the STFT, the second order LPFT, and the WVD, respectively. The horizontal axis is the normalized frequency, and the vertical axis is the normalized amplitude.

condition that the polynomial coefficients are accurately estimated. The effects of the window width, the estimation errors, and the order mismatch by using the second order LPFT to process higher-order PPSs are discussed. Resolution comparisons with the STFT and WVD are also provided to show the merits of the LPFT, and the LPFT is found to be a better tool to dealing with timevarying signals.

\section{Appendix}

Review on uncertainty principles of the STFT

This appendix provides an example of deriving the uncertainty principle for the STFT. The definitions of some terminologies in this appendix are also used in the main sections of this article. Let us consider the Pthorder PPS defined in (3) as the input signal. By multiplying $s(t)$ with a window function $h(t)$, the local signal is defined as $s_{t}(\tau)=s(\tau) h(t-\tau)$. It is easily seen that the Fourier transform of the local signal $s_{t}(\tau)$ is exactly the STFT of the original signal $s(t)$. The normalized local signal at time $t$ is

$$
\eta_{t}(\tau)=\frac{s(\tau) h(\tau-t)}{\sqrt{\int|s(\tau) h(\tau-t)|^{2} d \tau}},
$$

which ensures that for any $t, \int\left|\eta_{t}(\tau)\right|^{2} d \tau=1$. Let us review some terminologies based on the normalized local signal $\eta_{t}(\tau)$ [29] which is a function of $\tau$ within a short duration, and its Fourier transform is defined as

$$
F_{t}(\omega) \int \eta_{t}(\tau) \exp \{-j \omega \tau\} d \tau,
$$

to reveal the spectral information around the time instant $t$. For this normalized local signal, all the relevant quantities, such as the mean time duration, mean frequency and bandwidth, are time dependent [29].

The mean time of the normalized local signal is defined as

$$
\langle\tau\rangle_{t}=\int \tau\left|\eta_{t}(\tau)\right|^{2} d \tau=\frac{\int \tau|s(\tau) h(\tau-t)|^{2} d \tau}{\int|s(\tau) h(\tau-t)|^{2} d \tau},
$$


and its time duration is defined as

$$
T_{t}^{2} \int\left(\tau-\langle\tau\rangle_{t}\right)^{2}\left|\eta_{t}(\tau)\right|^{2} d \tau=\left\langle\tau^{2}\right\rangle_{t}-\langle\tau\rangle_{t}^{2},
$$

where $\left\langle\tau^{2}\right\rangle_{t}$ is the second order moment of the normalized local signal, defined as

$$
\left\langle\tau^{2}\right\rangle_{t}=\int \tau^{2}\left|\eta_{t}(\tau)\right|^{2} d \tau
$$

Similarly, the $n$th order moment of the normalized local signal is defined as

$$
\left\langle\tau^{n}\right\rangle_{t}=\int \tau^{n}\left|\eta_{t}(\tau)\right|^{2} d \tau
$$

The mean frequency of the normalized local signal is defined as

$$
\langle\omega\rangle_{t}=\int \omega\left|F_{t}(\omega)\right|^{2} d \omega=\int \eta_{t}^{*}(\tau) \frac{1}{j} \frac{d}{d \tau} \eta_{t}(\tau) d \tau,
$$

and its bandwidth is

$$
B_{t}^{2}=\int\left(\omega-\langle\omega\rangle_{t}\right)^{2}\left|F_{t}(\omega)\right|^{2} d \omega=\left\langle\omega^{2}\right\rangle_{t}-\langle\omega\rangle_{t}^{2},
$$

where

$$
\left\langle\omega^{2}\right\rangle_{t}=\int \omega^{2}\left|F_{t}(\omega)\right|^{2} d \omega=-\int \eta_{t}^{*}(\tau) \frac{d^{2} \eta_{t}(\tau)}{d \tau^{2}} d \tau
$$

Here the duration $T_{t}^{2}$ and bandwidth $B_{t}^{2}$ are good measures of the broadness of the local signal in the time and frequency domains, respectively. For example, $T_{t}^{2}$ (or $B_{t}^{2}$ ) of a signal means the width of the signal concentrated around the mean time $\langle\tau\rangle_{t}$ (or mean frequency $\left.\langle\omega\rangle_{t}\right)$. The uncertainty product in the STFT domain is generally a function of time, the signal $s(t)$ and the window $h(t)$, and has a lower bound [29]

$$
B_{t}^{2} T_{t}^{2} \geq \frac{1}{4}
$$

It should be noted that the focus of this article is on the uncertainty product obtained by multiplying the duration and bandwidth of the local signal. It is important to understand that this uncertainty product places limits on the processing techniques of the windowed transforms. Other kinds of uncertainty products, such as the global uncertainty products, can be referred to [17].

\section{Endnote}

${ }^{a}$ For simplicity in the rest of the article, the integral without limits implies that the integra-tion is from $-\infty$ to $\infty$.

\section{Abbreviations}

STFT: short-time Fourier transform; LPFT: local polynomial Fourier transform: WD: Wigner-Ville distribution; PPSs: polynomial phase signals; PTFT: polynomial time frequency transform; FFT: fast Fourier transform.

\section{Acknowledgements}

This research work was supported by the National Natural Science sFoundation of China (No. 61102164), and the Scientific Research Fund of Hangzhou Normal University (No. 2011QDL021).

\section{Author details}

${ }^{1}$ School of Information Science and Engineering, Hangzhou Normal University, China ${ }^{2}$ School of Electrical and Electronic Engineering, Nanyang Technological University, Singapore ${ }^{3}$ School of Electronic Information and Electrical Engineering, Shanghai Jiao Tong University, China

\section{Competing interests}

The authors declare that they have no competing interests.

Received: 9 September 2011 Accepted: 7 June 2012

Published: 7 June 2012

\section{References}

1. H Ozaktas, Z Zalevsky, M Kutay, The Fractional Fourier Transform With Applications in Optics and Signal Processing, Wiley, Chichester, (2001)

2. F Zhang, YQ Chen, G Bi, Adaptive harmonic fractional Fourier transform. IEEE Signal Process Lett. 6(11), 281-283 (1999)

3. V Katkovnik, A new form of Fourier transform for time-frequency estimation Signal Process. 47(2), 187-200 (1995). doi:10.1016/0165-1684(95)00107-7

4. I Djurovic, T Thayaparan, L Stankovic, Adaptive local polynomial Fourier transform in ISAR. EURASIP J Appl Signal Process. 2006, 1-15 (2006)

5. X Li, G Bi, Y Ju, Quantitative SNR analysis for ISAR imaging using local polynomial Fourier transform. IEEE Trans Aerosp Electron Syst. 45(3), 1241-1248 (2009)

6. M Dakovic, T Thayaparan, S Djukanovic, L Stankovic, Time-frequency-based non-stationary interference suppression for noise radar systems. IET Radar Sonar Navig. 2(4)، 306-314 (2008). doi:10.1049/iet-rsn:20070137

7. L Stankovic, S Djukanovic, Order adaptive local polynomial FT based interference rejection in spread spectrum communication systems. IEEE Trans Instrum Meas. 54(6), 2156-2162 (2005). doi:10.1109/TIM.2005.858567

8. V Katkovnic, A Gershman, A local polynomial approximation based beamforming for source localization and tracking in nonstationary environments. IEEE Signal Process Lett. 7(1), 3-5 (2000)

9. X Li, G Bi, S Stankovic, A Zoubir, Local polynomial Fourier transform: a review on recent development and applications. Signal Process. 91, 1370-1393 (2011). doi:10.1016/j.sigpro.2010.09.003

10. D Gabor, Theory of communication. J Inst Electr Eng. 93(26), 429-457 (1946)

11. P Korn, Some uncertainty principles for time-frequency transforms of the Cohen class. IEEE Trans Signal Process. 53(2), 523-527 (2005)

12. P Loughlin, L Cohen, The uncertainty principle: global, local, or both? IEEE Trans Signal Process. 52(5), 1218-1227 (2004)

13. S Shinde, V Gadre, An uncertainty principle for real signals in the fractional Fourier transform domain. IEEE Trans Signal Process. 49(11), 2545-2548 (2001). doi:10.1109/78.960402

14. D Mustard, Uncertainty principle invariant under fractional Fourier transform. J Austr Math Soc Ser B. 33, 180-191 (1991). doi:10.1017/ S0334270000006986

15. K Sharma, S Joshi, Uncertainty principle for real signals in the linear canonical transform domain. IEEE Trans Signal Process. 56(7), 2677-2683 (2008)

16. $\mathrm{GXu}, X$ Wang, $X X \mathrm{Xu}$, The logarithmic, Heisenberg's and short-time uncertainty principles associated with fractional Fourier transform. Signal Process. 89(3), 339-343 (2009). doi:10.1016/j.sigpro.2008.09.002

17. L Cohen, The uncertainty principles of the short-time Fourier transform. Proc SPIE. 2563, 80-90 (1995)

18. V Katkovnikm, Discrete-time local polynomial approximation of the instantaneous frequency. IEEE Trans Signal Process. 46(10), 2626-2637 (1998). doi:10.1109/78.720366 
19. $X \mathrm{Li}, \mathrm{GBB}$, Uncertainty principle of the second-order LPFT, in IEEE International Symposium on Circuits and Systems (ISCAS'2009), Taipei, Taiwan, 325-328 (24-27 Mat 2009)

20. M Ikram, K Abed-Meraim, Y Hua, Fast quadratic phase transform for estimating the parameters of multicomponent chirp signals. Dig Signal Process. 7(2), 127-135 (1997). doi:10.1006/dspr.1997.0286

21. X Xia, Discrete chirp-Fourier transform and its applications to chirp rate estimation. IEEE Trans Signal Process. 48(11), 3122-3133 (2000). doi:10.1109/ 78.875469

22. G Bi, Y Wei, Radix-2 DIF fast algorithms for polynomial time frequency transforms. IEEE Trans Aerosp Electron Syst. 42(4), 1540-1546 (2006)

23. $\mathrm{Y} \mathrm{Ju}, \mathrm{G} \mathrm{Bi}$, Generalized fast algorithms for the polynomial time-frequency transform. IEEE Trans Signal Process. 55(10), 4907-4915 (2007)

24. $\mathrm{GBi}, \mathrm{Y}$ Chen, Fast DFT algorithms for length $N=q^{*} 2^{m}$. IEEE Trans Circ Syst II-Analogu Dig Signal Process. 45(6), 685-690 (1998). doi:10.1109/ 82.686687

25. G Bi, Y Chen, Y Zeng, Fast algorithms for generalized discrete Hartley transform of composite sequence lengths. IEEE Trans Circ Syst II-Analogu Dig Signal Process. 47(9), 893-901 (2000). doi:10.1109/82.868457

26. $\mathrm{G} \mathrm{Bi}, \mathrm{Y}$ Chen, Fast generalized DFT and DHT algorithms. Signal Process. 65(3), 383-390 (1998). doi:10.1016/S0165-1684(97)00234-X

27. I Djurovic, Robust adaptive local polynomial Fourier transform. IEEE Signal Process Lett.11(2), 201-204 (2004)

28. Y Wei, G Bi, Efficient analysis of time-varying muliticomponent signals with modified LPTFT. EURASIP J Appl Signal Process. 2005(1), 1261-1268 (2005)

29. L Cohen, Time-Frequency Analysis, (Prentice Hall, Englewood Cliffs, NJ, 1995)

doi:10.1186/1687-6180-2012-120

Cite this article as: $L i$ et al:: On uncertainty principle of the local polynomial Fourier transform. EURASIP Journal on Advances in Signal Processing 2012 2012:120.

\section{Submit your manuscript to a SpringerOpen ${ }^{\circ}$ journal and benefit from:}

- Convenient online submission

- Rigorous peer review

- Immediate publication on acceptance

- Open access: articles freely available online

- High visibility within the field

- Retaining the copyright to your article

Submit your next manuscript at $\gg$ springeropen.com 